\title{
Position of alveolodental frasments before and after primary gingivoperiosteoplasty in unilateral cleft lip and palate
}

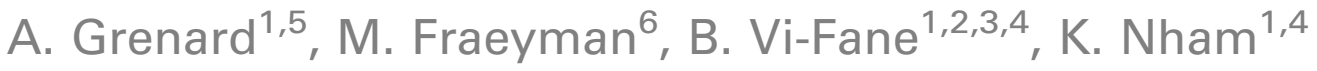 \\ 1 Dental surgeon \\ 2 Dentofacial orthopedics specialist \\ 3 University lecturer - Consultant \\ 4 MAFACE rare facial diseases reference center \\ 5 CECSMO orthodontic certification studies (4 $4^{\text {th }}$ year) \\ 6 Postgraduate dentofacial orthopedics certificate (DES ODF) $\left(2^{\text {nd }}\right.$ year)
}

\section{ABSTRACT}

The orofacial clefts are the most frequently facial congenital malformations in the world (1 birth per 700). There are a lot of protocols which are different in the management of timetables and surgical techniques.

The aim of this work is to study the tridimensional evolution between two alveolodental fragments during orthodontic surgery cares of patients with unilateral FLAP in the center MAFACE (maxillo-facial and plastic surgery unit at Necker's hospital and rare diseases unit at Rothschild's hospital).

The first step of orthodontic care is to make a transversal expansion of maxillary in order to obtain an optimal width before gingiviperiostoplasty (GPP) with bone grafting.

We have studied the tridimensional radiographic documents at different times: TO (initial time before orthodontic treatment and GPP), T1 (after the orthodontic expansion and before GPP) and T2 (six months after GPP).

\section{KEY WORDS}

Orthodontics, unilateral cleft lip and palate, gingivoperiosteoplasty

\section{INTRODUCTION}

Cleft lip and palate is the most frequent congenital facial deformity worldwide, affecting 1 in 700 live births in France $2,5,17$.
Treatment should enable social and familial integration. It comprises improved facial morphology, and restoration 
of function (notably, phonatory and auditory), while avoiding any procedure liable to impair growth. It requires multidisciplinary team-work.

There are a wide variety of treatment protocols. In 2001, the Eurocleft association found 201 teams treating CLP, with 194 different protocols ${ }^{14}$, differing in treatment schedule and surgical technique. Pr Vazquez's team in the

\section{UNILATERAL CLEFT LIP AND PALATE}

Cleft lip and palate (CLP) results from persistence of embryonic clefts which normally, but temporarily, separate the nasal and maxillary buds. This fusion defect occurs between the $5^{\text {th }}$ and $8^{\text {th }}$ week of development, and may be uni- or bi-lateral, complete or incomplete ${ }^{1}$.

Unilateral CLP follows the philtral crest then continues and separates the alveolar crest at the lateral incisor. Beyond the anterior palatine canal, it becomes medial, dividing the hard and soft palates ${ }^{4}$.

Patients show arciform distortion of the face (Fig. $1 \mathrm{a}, \mathrm{b}, \mathrm{c}$ ). The nasal spine is shifted away from the healthy side and the chin from the cleft side. The tongue is hypoplastic, with a cutaneo-mucosal line and mucosal part running obliquely toward the nasal sill ${ }^{15}$.

The nose is distended and deformed. On the affected side, the nasal wing is stretched transversally. The inferior nasal septum and base of the columella are shifted toward maxillofacial and plastic surgery department of the Necker Hospital (Paris, France), formerly based in the Trousseau Hospital, performs first-line gingivoperiosteoplasty following Talmant.

The present study describes the $3 \mathrm{D}$ evolution of the two alveolodental fragments during orthodontic surgical treatment of patients managed from birth in this department.

the healthy side ${ }^{8}$. The nasal floor is absent on the affected side.

The superior facial bones are divided into two fragments:

- One large fragment, supporting the nasal septum, incisor block and anterior nasal spine; muscle pressure and septal growth tend to push this fragment forward and outward;

- A smaller fragment, showing little displacement at birth but tending to shift inward under nasal pyramid pressure in the absence of counter-pressure from the tongue ${ }^{8}$.

Unilateral CLP has repercussions for facial bone growth due to repair surgery and induces functional disorders: oral respiration and infantile swallowing disorder. There is often vertical contraction of the maxillary arcade, with endognathy. Pressure from the upper lip exacerbates the facial asymmetry.

Dental abnormalities are frequently associated. The most common is agenesis of the lateral incisor on the 
cleft side ${ }^{16}$. Other abnormalities are also frequently reported: microdontia ${ }^{11}$ or a double lateral incisor ${ }^{16}$. There may also be agenesis of the second premolars, structural abnormalities (enamel hypoplasia) ${ }^{3}$ and delayed root construction? ${ }^{7}$.

\section{CLP MANAGEMENT IN THE NECKER HOSPITAL MAXILLOFACIAL SURGERY DEPARTMENT ${ }^{10}$}

CLP treatment is long-term, from birth to adulthood. Management is multidisciplinary, and begins with diagnosis: i.e., even before birth. The entire treatment plan should be determined in advance and reassessed step by step.

\section{Prenatal care}

CLP can be diagnosed from the second trimester ultrasound scan. The prenatal diagnosis is then discussed in a meeting of the multidisciplinary prenatal diagnosis center. An antenatal surgery consultation provides the opportunity to explain matters to the parents and prepare them for the global treatment plan.

\section{Pre-surgical orthopedics}

If the infant presents feeding disorder, a palatine obturator plate is fitted at birth, before primary surgery.

\section{Surgery: step 1}

Surgery is performed at 3 months and comprises 3 procedures:

- Intravelar veloplasty, to reconstruct the muscle strap of the levator veli palatini;

- Cheiloplasty, to normalize philtral crest height, restore lip symmetry with respect to the healthy side and restore the Cupid's bow;

- Rhinoplasty, to reposition the alar cartilage and nasal septum.

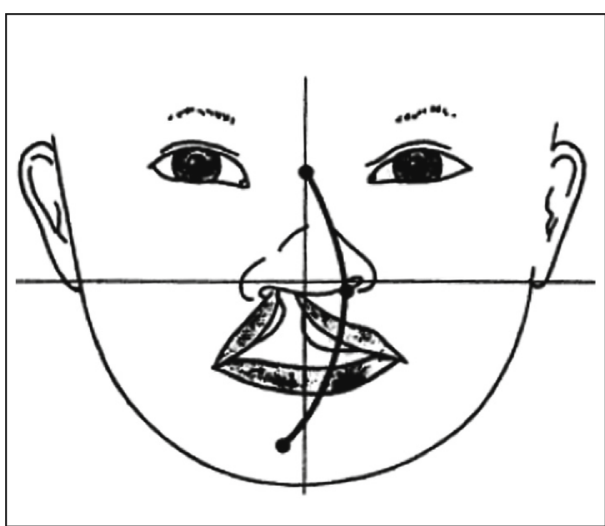

a

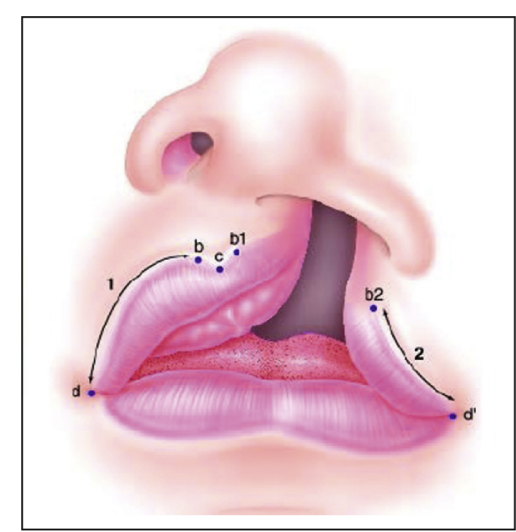

b

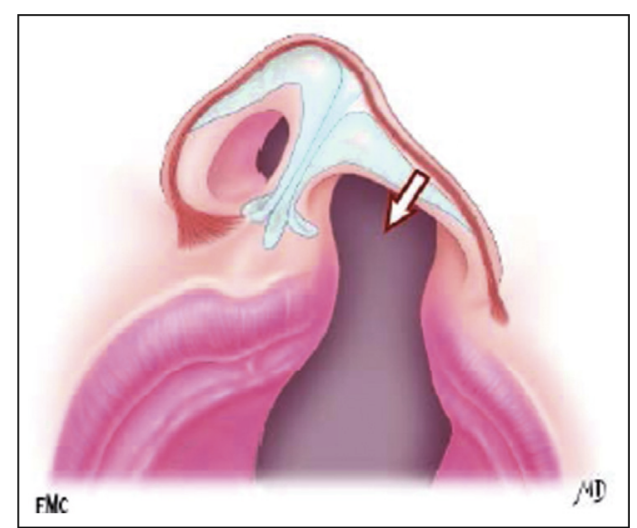

c

Figure 1

(a) Arciform facial view, taken from Malek ${ }^{8}$. (b) AP lip view, from Pavy 9 . (c) Inferior alar cartilage view, from Pavy ${ }^{9}$ 


\section{Surgery: step 2}

Bony palate closure is performed about 12 months after step 1, as the hard palate cleft shrinks spontaneously after surgical closure of the lip and soft palate.

\section{Surgery: step 3}

Gingivoperiosteoplasty (GPP) is performed at 4-6 years. An iliac cancellous bone graft is performed up the entire maxilla to the piriform orifice. This is the final primary step in cleft closure. It usually follows a period of orthodontic preparation to achieve transverse expansion to correct endognathy, especially in the smaller fragment. The preparation seeks to obtain an inter-canine distance (CC') of $32-35 \mathrm{~mm}$ ahead of GPP. Expansion preferably uses a Quad-Helix expander on a dental guard or, exceptionally, on a removable plate with medial jack. The patient is fitted with a removable Quad-Helix appliance mounted on sheaths of temporary rings fixed to the second molars ahead of GPP. The Quad-Helix will be removed during surgery then repositioned and left in contention for 6-9 months.

\section{Follow-up}

\section{Orthodontic follow-up}

In mixed denture, follow-up basically consists in monitoring the evolution of the permanent incisors, adjusting space, correcting rotation and treating anterior occlusion inversion. In young adult denture, orthodontic treatment is more or less classical, completed by orthognathic surgery if the discrepancy between the bone foundations is too great. The objective is to conserve a normal sized maxillary arcade, not closing the space of the lateral incisor if it is missing. Implant-borne rehabilitation or a prosthesis in extension (Cantilever) are the preferred solutions at end of growth.

\section{Surgical follow-up}

Surgery may be performed at end of growth (clefts being frequently associated with a class III skeletal pattern due to retromaxillism) or consist in correction of sequelae (lip, nose or palate deformity or fistula closure, etc.). The surgical decision is made on demand in a multidisciplinary meeting.

\section{ENT follow-up}

Yearly auditory screening is systematic as of 9 months.

\section{Speech-therapy follow-up}

Treatment within the department with a specialized speech-therapist is initiated early. The objective is to make the parents aware of the importance of the prephonatory phase and the possibility of velopharyngeal rehabilitation. Early speech-therapy in CLP seeks notably to optimize phonation to enable language acquisition before primary school. 


\section{Objectives}

The aim of the present study was to describe the 3-dimensional evolution of the two alveolodental fragments during orthodontic surgical treatment of unilateral CLP in the Rare Diseases Department of the Necker Hospital (Paris, France). Does it resemble slow expansion by maxillary disjunction in the absence of palatine suture?

We also looked for improvement in or correction of the horizontal and vertical positions of the fragments with respect to the occlusion plane after orthodontic expansion, and the durability of results after GPP.

\section{Material}

\section{Sample}

The sample comprised 16 patients undergoing treatment for CLP in $\mathrm{Pr}$ Vasquez's maxillofacial and plastic surgery department in the Necker Hospital of Paris (France). The presented unilateral CLP and were aged between 3 and 5 years. They underwent preparatory orthodontic transverse expansion, performed by Dr Vi-Fane's team in private practice or in $\mathrm{Pr}$ Berdal's rare diseases reference center and in $\operatorname{Pr}$ M.L. Boy-Lefèvre's functional unit in the Rothschild Hospital. The preparation was followed by primary GPP performed in the maxillofacial surgery department of the Trousseau Hospital.

\section{Radiology}

The study was based on comparative analysis of CT scans taken at 3 time-points for each patient:

- TO: baseline CT or cone-beam CT (CBCT) scan before orthodontics and GPP;

- T1: second CT or CBCT scan after orthodontic expansion and just before GPP;

- T2: third CT or CBCT scan, 6 months after GPP and just before or after removal of the QuadHelix appliance.

These examinations were prescribed by the surgeon or orthodontist for patient management rather than for the study as such; observation was thus retrospective.

\section{Data measurement and representation}

Observation and angular and metric measurement used the opensource version of the Osiri ${ }^{\circledR}$ medical imaging software, with native DiCOM slices. Graphs were created on KaléidaGraph ${ }^{\circledR}$ software.

\section{Method}

\section{Determination of reference axes}

The posterior reference axis was determined on an axial slice from the line through the two palatine foramens, and corresponded to the $\mathrm{X}$-axis of the graphs. The median of 
the $X$ segment thus constituted the patient's theoretic sagittal plane.

Frontally, the superior plane was determined arbitrarily by the palatine plane (Z).

\section{Determination of anatomic points}

All measurements were made by the same operator after a running-in time to recognize anatomic structures on deciduous and permanent canines and deciduous second molars, in both fragments.

For each tooth, the following were located on axial slices:

- An apical point: apex of the deciduous canines, tip of the palatine root for the deciduous molar; the permanent canines do not need measuring as root construction has not started;

\section{RESULTS}

The KaléidaGraph ${ }^{\circledR}$ software presented results with orthonormal landmarks:

- $\mathrm{X}$ : posterior transverse dimension;

- $Y$ : theoretic sagittal axis;

- Z: theoretic palatine plane.

For each patient, an axial and AP representation of the teeth could thus be obtained (Fig. 3 a, b).

Figure 3 is a KaléidaGraph ${ }^{\circledR}$ representation of the deciduous molars on axial (3a) and AP view (3b) in patient $n^{\circ} 16$ (right CLP).

Thus, for the deciduous molars, between T0 (baseline) and T1 (end of
- An alveolar plus coronary point: tip of the deciduous and permanent canines, deciduous second molar furcation (note: cuspid abrasion is common and may induce error);

Finally, an inter-incisor point was also located for each patient: center of the space between the two permanent incisors.

The slice thickness between the points and the palatine plane represents the height of the anatomic elements.

The measurements were reproduced, depending on the records available, at T0, T1 and T2 for all 16 patients. The whole data-set constituted polar coordinates that could be transcribed on AP and axial views to quantify evolution and visualize alveolodental movement (Fig. 2).

orthodontic preparation and before GPP) there was:

- 4.5-mm transverse increase by vestibular version, in the small fragment only;

- No change in vertical difference between the fragments; the occlusion plane remained inclined toward the large fragment.

\section{Discussion}

The results as a whole can be represented by the behavioral trends between T0 and T2 (Fig. 4) and between T1 and T2 (Fig. 5). 


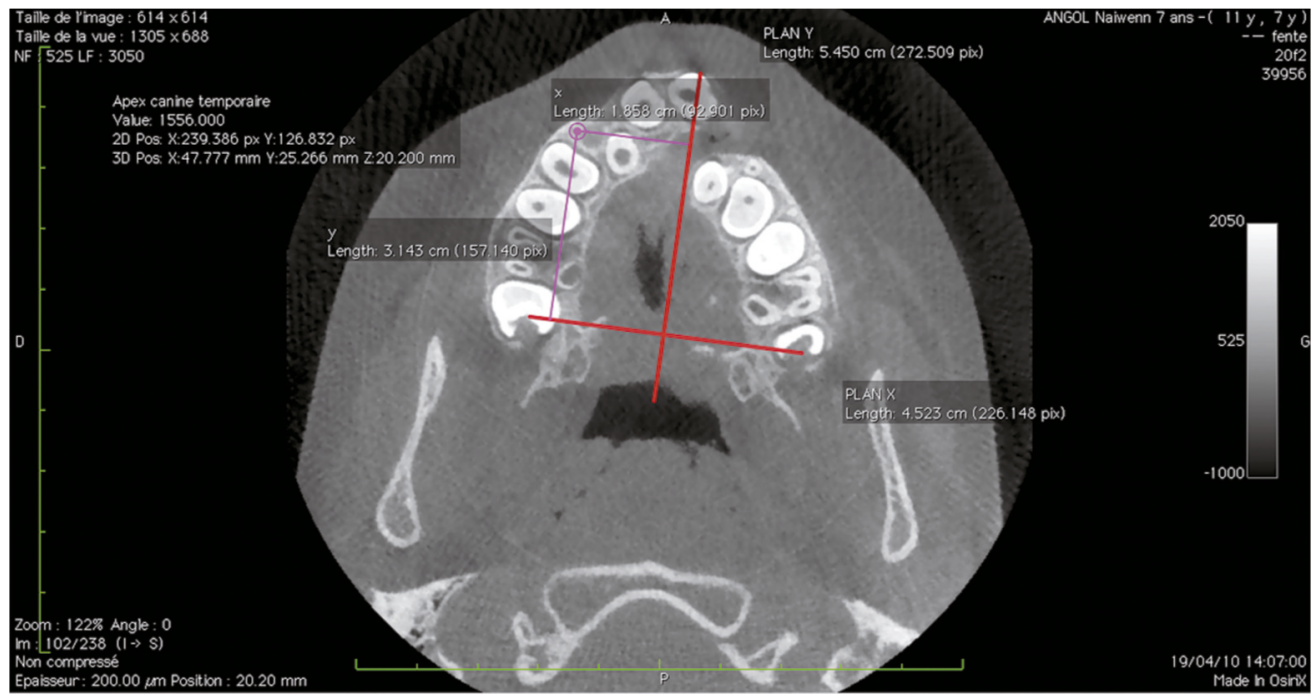

Figure 2

Right permanent canine tip with polar coordinates.
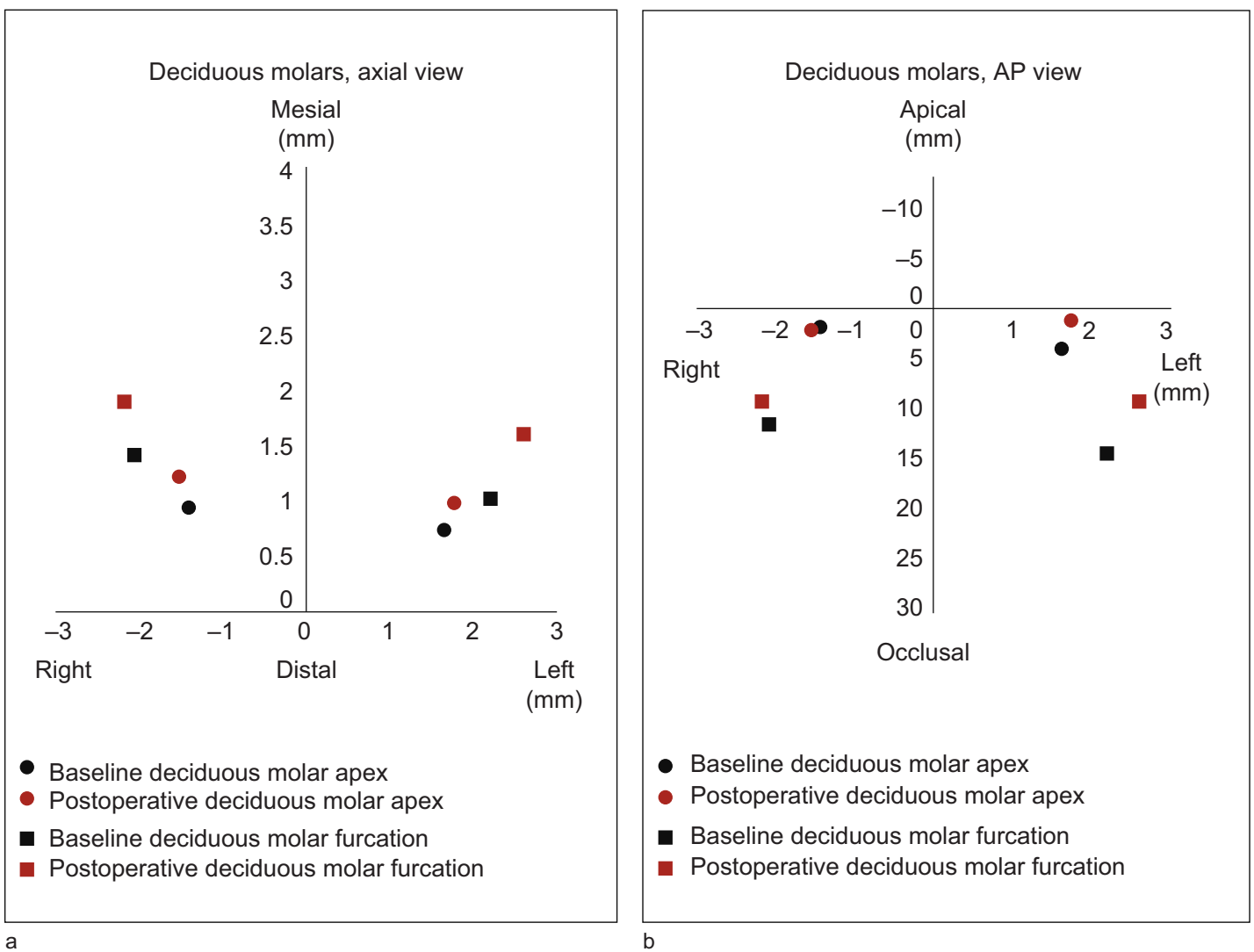

Figure 3

KaléidaGraph ${ }^{\circledR}$ representations of deciduous molars, axial (a) and AP view (b), at T0 and T2 for patient $n^{\circ} 16$ (right CLP). 


\begin{tabular}{|c|c|c|c|c|c|c|c|}
\hline \multirow{2}{*}{ PATIENTS } & \multicolumn{2}{|c|}{ Deciduous canines } & \multicolumn{2}{|c|}{ Definitive canines } & \multicolumn{2}{|c|}{ Deciduous molars } & \multirow{2}{*}{$\begin{array}{c}\text { Incisor center } \\
\text { Correction } \\
\text { of incisor } \\
\text { center }\end{array}$} \\
\hline & $\begin{array}{l}\text { Transverse } \\
\text { dimension }\end{array}$ & $\begin{array}{l}\text { Shift between } \\
2 \text { fragments }\end{array}$ & $\begin{array}{l}\text { Transverse } \\
\text { dimension }\end{array}$ & $\begin{array}{l}\text { Shift between } \\
2 \text { fragments }\end{array}$ & $\begin{array}{l}\text { Transverse } \\
\text { dimension }\end{array}$ & $\begin{array}{l}\text { Shift between } \\
2 \text { fragments }\end{array}$ & \\
\hline Pat 11 & $\pi$ & $\pi$ & 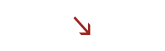 & $\pi$ & - & - & yes \\
\hline Pat 12 & $\pi$ & 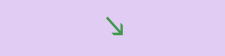 & $\rightarrow$ & $\lambda$ & $\lambda$ & $\searrow$ & yes \\
\hline Pat 13 & $\lambda$ & $\lambda$ & $\lambda$ & $\pi$ & $\lambda$ & $\rightarrow$ & no \\
\hline Pat 14 & $\rightarrow$ & $\lambda$ & $\pi$ & $\pi$ & $\rightarrow$ & 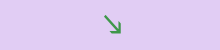 & $\rightarrow$ \\
\hline Pat 15 & $\pi$ & 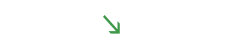 & $\rightarrow$ & $\rightarrow$ & $\pi$ & 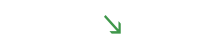 & $\rightarrow$ \\
\hline Pat 16 & $\lambda$ & $\searrow$ & $\rightarrow$ & $\downarrow$ & $\lambda$ & $\checkmark$ & $\rightarrow$ \\
\hline
\end{tabular}

Figure 4

Evolution between T0 and T2. Green shows improvement, red aggravation, $\nearrow$ increase, and $\searrow$ decrease.

\begin{tabular}{|c|c|c|c|c|c|c|c|}
\hline \multirow{2}{*}{ PATIENTS } & \multicolumn{2}{|c|}{ Deciduous canines } & \multicolumn{2}{|c|}{ Definitive canines } & \multicolumn{2}{|c|}{ Deciduous molars } & \multirow{2}{*}{\begin{tabular}{|c|} 
Incisor center \\
$\begin{array}{c}\text { Correction } \\
\text { of incisor } \\
\text { center }\end{array}$
\end{tabular}} \\
\hline & $\begin{array}{l}\text { Transverse } \\
\text { dimension }\end{array}$ & $\begin{array}{l}\text { Shift between } \\
2 \text { fragments }\end{array}$ & $\begin{array}{l}\text { Transverse } \\
\text { dimension }\end{array}$ & $\begin{array}{l}\text { Shift between } \\
2 \text { fragments }\end{array}$ & $\begin{array}{l}\text { Transverse } \\
\text { dimension }\end{array}$ & $\begin{array}{l}\text { Shift between } \\
2 \text { fragments }\end{array}$ & \\
\hline Pat 2 & $\rightarrow$ & ע & $\rightarrow$ & ע & $\lambda$ & ע & $\rightarrow$ \\
\hline Pat 3 & $\rightarrow$ & $\searrow$ & $\rightarrow$ & $\rightarrow$ & $\rightarrow$ & $\rightarrow$ & $\rightarrow$ \\
\hline Pat 4 & $\rightarrow$ & ע & $\rightarrow$ & 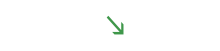 & $\lambda$ & $\searrow$ & yes \\
\hline Pat 5 & $\rightarrow$ & $\pi$ & $\rightarrow$ & $\rightarrow$ & $\rightarrow$ & $\rightarrow$ & $\rightarrow$ \\
\hline Pat 6 & ע & ע & y & $\rightarrow$ & $\rightarrow$ & $\pi$ & yes \\
\hline Pat 7 & $\pi$ & $\rightarrow$ & $\searrow$ & $\rightarrow$ & ע & $\searrow$ & no \\
\hline Pat 8 & $\rightarrow$ & $\rightarrow$ & ע & $\pi$ & $\lambda$ & $\rightarrow$ & yes \\
\hline Pat 9 & $\rightarrow$ & $\lambda$ & $\rightarrow$ & $\lambda$ & $\rightarrow$ & $\pi$ & yes \\
\hline Pat 10 & $\rightarrow$ & $\searrow$ & $\lambda$ & $\searrow$ & $\rightarrow$ & $\lambda$ & yes \\
\hline
\end{tabular}

Figure 5

Evolution between T1 and T2. Green shows improvement, red aggravation, 7 increase, and $\searrow$ decrease. 


\section{Movements obtained by orthodontic preparation}

The results seem to show transverse increase at the end of orthodontic preparation, more anterior than posterior, in agreement with other reports $^{6,13}$. Expansion is by coronary vestibular version of the small fragment at the deciduous canines and molars. The transverse dimension, in contrast, is stable at the permanent canines. These movements are of exclusively alveolodental origin.

\section{Movements obtained after surgery}

The transverse dimension seems stable at the deciduous and permanent canines. At the deciduous molars, it also seems stable, or slightly increased; this may be due to intraoperative repositioning of the QuadHelix appliance after GPP, creating transverse contention. The appliance thus maintains the transverse dimension at the canines and deciduous molars. It may sometimes be reactivated on request, explaining some cases of further increase in the transverse dimension after GPP.

\section{Medium-term results}

The transverse dimension is finally greater at T2 than at baseline (TO), at both the deciduous canines and deciduous molars. Orthodontic preparation thus provides direct benefit.

Expansion is alveolodental. There is coronary vestibular version in the large but also in the small fragment, although of larger amplitude in the large fragment in some patients.
The transverse dimension at the permanent canines is relatively stable or slightly increased. There would thus seem to be little basal action during treatment.

\section{Study limitations}

\section{Sample}

Sample size precluded general conclusions as serious statistical analysis was not possible. This limitation can be explained by several factors:

- Patients were included only if managed in the MAFACE reference center or by Dr Vi-Fane in private practice and treated by $\mathrm{Pr}$ Vasquez's team following the 2000 protocol from primary surgery onward;

- As radiology documents were used on several sites, data collection was more difficult, priority obviously being given to surgery;

- The radiology documents were required to be native DiCOM files so as to be able to be analyzed in three dimensions by the OsiriX ${ }^{\circledR}$ software.

\section{Anatomic landmarks}

Despite all our efforts to ensure rigorous location of the anatomic units, some biases may have occurred in observation and measurement:

- Deciduous second molar root resorption may make the determination of apical points less precise, but is moderate in this phase;

- Measurements were all taken by a single observer, but intra-observer 
variability was not tested by repeated measurement;

- Even so, the observer complied with a running-in time for recognizing anatomic structures on Osirix ${ }^{\circledR}$ before study start-up; structures were checked by two other observers, one of whom was experienced in radiologic anatomy;

- The posterior plane between the foramens is considered stable between the ages of 4 and 6 years (the study age-bracket); the median, less subject to error, was used in determining sagittal references.

\section{CONCLUSION}

With global patient management, primary surgery and a low rate of revision surgery, the unilateral CLP treatment schedule implemented by Pr Vasquez's team provides a more functional approach, limiting the scarring which impairs maxillary growth.

CLP treatment consists in increasing the transverse dimension of the maxillary arcade (especially the intercanine distance) to an optimal degree before GPP, and maintaining it in contention or further increasing it if necessary.

Although the sample was small, the present preliminary results confirm the alveolodental origin of expansion, exclusively driven by coronary vestibular version:

- in the smaller fragment during orthodontic preparation;

- and in both fragments after GPP.

Movement amplitude was greater in the anterior than the posterior

\section{Radiology documents}

The three radiologic series were not all performed by the same radiologist, and equipment differed. Most images were on CBCT, using algorithms to transcribe density as gray value, creating a scale analogous to CT Hounsfield units. This scale varied between machines and settings. However, the study focused on linear and angular measurements and anatomic landmarks, and not on graft density or volume, so that this difference in equipment did not affect results.

region, as classically reported in rapid maxillary expansion, along with alveolodental compensation.

The classical recurrence associated with maxillary expansion was less frequent, due to the observation time, final radiology being performed with the Quad-Helix still in place or only recently removed.

The present preliminary results encourage us to continue the study on a larger scale and longer period. Our sample size can only increase with time. Continued follow-up, moreover, will enable the variable of growth to be included, with possible recurrence in the transverse dimension in the medium-term (2 years) after removal of apparatuses, and also monitoring of the evolution of the permanent canines, usually facilitated by the constitution of a continuous arcade.

Finally, standardizing the CBCT protocol between two sites will enable 
the radiology documents to be formatted, adding a new dimension to the study: graft density and integration $^{12}$.
Conflicts of interest: The author declares no conflict of interest.

\section{BIBLIOGRAPHY}

1. Bath-Balogh M, Fehrenbach MJ, Thomas P. Illustrated dental embryology, histology, and anatomy. St. Louis, Mo.: Elsevier Saunders, 2006.

2. Bender PL. Genetics of cleft lip and palate. J Pediatr Nurs 2000;15(4):242-9.

3. Camporesi M, Baccetti T. Maxillary dental anomalies in children with cleft lip and palate: a controlled study. Int J Paediatr Dent 2010;20(6):442-50.

4. Derbal A. Diagnostic clinique et étude du sens transversaldes arcades dentaires des porteurs de fentes labio-palatines unilatérales. Université Bordeaux 2-Victor Segalen, 2003.

5. Hohlfeld J. Les fentes labio-maxillo-palatines. Journal de pédiatrie et de puériculture 1991;8(4):459-63.

6. Isaacson RJ, Murphy TD. Some effects of rapid maxillary expansion in cleft lip and palate patients. Angle Orthod 1964;34(3).

7. Lai MC, King NM, Wong HM. Abnormalities of Maxillary Anterior Teeth in Chinese Children With Cleft Lip and Palate. The Cleft Palate Craniofac J 2009;46(1):58-64.

8. Malek R. Psaume J. Bases anatomiques et physiologiques du traitement primaire des fentes labio-alvéolo palatines unilatérales totales. AOS 1998;162:1988

9. Pavy $V$, Vacher $C$, Vendroux J. Fente labiale et palatine : traitement primaire. EMC Techniques chirurgicales - chirurgie plastique reconstructive et esthétique 1998; 45-580:1-21.

10. Picard $A$, et al. Calendrier et protocole interventionnel des fentes labio-palatines au sein du service de chirurgie maxillo-faciale et plastique de l'hôpital d'enfants Trousseau (AP-HP, Paris). Rev Orthop Dento Facial 2011;45(3):291-9.

11. Rakotoarison RA, et al. Étude des anomalies dentaires associées aux fentes labiopalatines: à propos de 85 cas. Med Buccale Chir Buccale 2011;17:7-14.

12. Rebiffé C. Protocole d'évaluation quantitative et qualitative des greffons osseux lors des gingivo-périostoplasties chez les enfants porteurs de fentes labioalvéolopalatines. Th Chir dent Paris 7, 2014.

13. Sandikciolu M, Hazar S. Skeletal and dental changesafter maxillary expansion in the mixed dentition. Am J Orthod Dent Orthop 1997;111(3):321-7.

14. Shaw WC, et al. (2001) The Eurocleft project 1996-2000: overview. J Craniomaxillofac Surg 29(3):131-140; discussion 141-142.

15. Tessier P. Anatomical classification of facial, cranio-facialand latero-facial clefts. J Maxillofac Surg 1976;4:69-92.

16. Tortora C, Meazzini MC. Prevalence of abnormalities in dental structure, position and eruption pattern in a population of unilateral and bilateral cleftlip and palate patients. Cleft Palate Craniofac J 2008;45(2):154-62.

17. Vanderas AP. Incidence of cleft lip, cleft palate, and cleft lip and palate among races: a review. Cleft Palate J 1987;24(3):216-25. 